Revue d'histoire de l'Amérique française

REVUE D.HISTOIRE DE L'AMÉRIQUE FRANÇAISE

\title{
La part du P. Claude Allouez dans les "Sentiments " qui lui sont attribués
}

\section{Léon Pouliot}

Volume 15, numéro 3, décembre 1961

URI : https://id.erudit.org/iderudit/302135ar

DOI : https://doi.org/10.7202/302135ar

Aller au sommaire du numéro

Éditeur(s)

Institut d'histoire de l'Amérique française

ISSN

0035-2357 (imprimé)

1492-1383 (numérique)

Découvrir la revue

Citer cet article

Pouliot, L. (1961). La part du P. Claude Allouez dans les « Sentiments » qui lui sont attribués. Revue d'histoire de l'Amérique française, 15(3), 379-395.

https://doi.org/10.7202/302135ar d'utilisation que vous pouvez consulter en ligne.

https://apropos.erudit.org/fr/usagers/politique-dutilisation/ 


\section{LA PART DU P. CLAUDE ALLOUEZ DANS LES «SENTIMENTS 》 QUI LUI SONT ATTRIBUÉS}

Nous présentons ici quelques constatations que nous avons faites en préparant, pour le Dictionnaire Biographique du Canada, la notice du P. Claude Allouez. Elles ne sont pas sans intérêt. Mais d'abord introduisons le personnage.

\section{LE P. CLAUDE ALLOUEZ (1622-1689)}

Né en Languedoc, à Saint-Didier-en-Forest, le 6 juin 1622, Claude Allouez fait ses études au collège des Jésuites du Puy ${ }^{1}$ et, à l'âge de 17 ans il entre au noviciat de Toulouse. Il arrive à Québec en même temps que le gouverneur d'Argenson, le 11 juillet $1658^{2}$; il est d'abord attaché aux résidences de Québec et des Trois-Rivières, tout en se livrant à l'étude de l'algonquin et du huron.

En 1663, il est nommé par Mgr de Laval grand vicaire pour les missions de l'ouest, premier pas vers l'organisation de l'Église dans ce qui est maintenant le centre des États-Unis. Le 8 août 1665 , il se met en route pour la région des Grands Lacs, où vivaient les nations dites outaouaises. Il s'y dépensa inlassablement pendant 24 ans. On aura une idée de ses travaux en considérant qu'il a sillonné en tous sens la région des Grands Lacs: Huron, Supérieur, Michigan, Érié. 3000 milles de pays sauvage, où ses ouailles se trouvaient dispersées et errantes; vingt-trois nations de races, de langues et de mœurs différentes, au dire du P. Dablon, son supérieur; il aurait baptisé de sa

1 Dans la Notice du P. Allouez, que nous devons au P. Dablon, on lit: - Ce fut dans cette dernière ville [Le Puy] qu'il reçut les prémices de l'esprit catholique par les communications qu'il eut avec le Père Francois Régis, de sainte mémoire, ayant souvent assisté, répondu et déclamé au catéchisme de ce fameux missionnaire. Là il fut appelé à notre Compagnie. 》 Margry, Découvertes et Etablissements des Français, I: 59. - Il se trouve que saint François Régis avait demandé les missions du Canada. Voir Georges Guitton, S.J., Saint Jean François Régis (Paris, 1937), 237-242 et 279-285.

2 Journal des Jésuites (Québec, 1871). 
main quelque 10,000 néophytes ${ }^{3}$. Le P. Gilbert Garraghan a pu écrire avec raison :

Dans l'Etat du Wisconsin, les premiers travaux missionnaires se rattachent à la personne du P. Claude Allouez. A la baie Chequamegon, près du moderne Ashland, et en maints endroits de l'intérieur, il a établi des postes de mission, qui sont devenus comme autant de centres de rayonnement de la civilisation chrétienne. [....] Nous lui devons nos plus anciennes connaissances sur les peuples Illinois. Si le nom de Jacques Marquette occupe une place exceptionnelle par son vibrant appel au sentiment et à l'imagination historique, Claude Allouez mérite d'être vénéré comme celui qui, le premier, implanta le catholicisme dans ce qui est maintenant le cœur des Etats-Unis. ${ }^{4}$

Les États-Unis, celui du Wisconsin en particulier, ont toujours honoré la mémoire du P. Allouez. En 1899, la Société Historique $\mathrm{du}$ Wisconsin lui élevait un monument à De Pere. Et cette Société avait alors pour secrétaire Reuben Gold Thwaites, le très méritant compilateur de la collection The Jesuit Relations and allied documents.

\section{LES « SENTIMENTS 》DU P. ALLOUEZ}

Le 29 août 1690, le P. Claude Dablon, supérieur des Jésuites de Québec ${ }^{5}$, adressait au Provincial de Paris une lettre qui n'a pas échappé à l'attention de Pierre Margry ${ }^{6}$. Elle commençait par ces mots :

Nos Pères qui sont chez les Outaouais nous ont appris la mort du Père Claude Allouez. Ils ont trouvé plusieurs écrits de ce cher défunt, lesquels sont pleins de l'esprit apostolique dont il était animé. Mais comme ils ne nous ont encore envoyé qu'une partie,

3 Margry, loc. cit.

4 The Jesuits in the Middle United States (New York, 1938), I: 3-4.

5 Le P. Dablon était un homme expéditif. Le 28 août 1690, il apprend la mort du P. Allouez, survenue exactement un an plus tôt, chez les Miamis, près de Niles (Michigan). Dès le lendemain, 29 août, il envoyait au Provincial de Paris la Notice du P. Allouez et, en un document distinct, la copie des Sentiments qui font l'objet de cette étude. graphe.

6 Texte dans Margry, op. cit., 64-72. Nous avons modernisé l'ortho- 
où ce grand missionnaire exprime ses sentiments sur sa vocation au Canada, sur les missions que nous y avons, et sur les missionnaires qu'il y faut, je ne puis faire part à V.R. que du peu que j'ai reçu. $\mathrm{Si}$ elle agrée le présent que je lui en fais dans cette fidèle copie, je prendrai la liberté de lui en faire un nouveau l'année prochaine, qu'on doit m'envoyer ce qui reste des papiers d'un si saint homme. ${ }^{7}$

Ainsi, ce que le P. Dablon transmet au Provincial de Paris, c'est la copie fidèle d'un document écrit tout entier de la main du P. Allouez. Il semble même que les titres des trois paragraphes qu'il contient et l'ordre dans lequel les phrases se suivent soient également du P. Allouez, le P. Dablon se contentant d'ajouter après le mot Sentiments les mots du Père Claude Allouez. Le P. Dablon concluait ainsi sa lettre au Provincial de Paris:

Voilà, mon R. Père, comment le feu Père Claude Allouez, de sainte mémoire, s'est, sans y penser, dépeint lui-même par ses propres paroles, qu'il n'avait écrites que pour sa consolation particulière et desquelles Dieu voudra peut-être bien se servir pour lui donner de dignes imitateurs. Ainsi soit-il.

Sans ombre d'hésitation, le P. Dablon attribue tout le document au P. Allouez. Sur quoi se base-t-il ? Sur l'écriture de son confrère et ami qui lui était connue. De plus, le contenu du document lui apparaissait, il nous le dit lui-même, comme le portrait de la grande âme et du grand cœur du P. Allouez. Mais avait-il raison?

\section{ANALYSE DES 《SENTIMENTS 》}

Le premier paragraphe a pour titre: «Sentiments du P. Claude Allouez sur sa vocation aux missions». Il appartient tout entier au P. Allouez. Celui-ci a fixé sur le papier, mais pour lui seul, les sentiments qu'il éprouvait dans son âme quand le 2 mars 1657, il recevait la grande grâce de sa vie: l'appel aux missions du Canada. Les deux autres paragraphes sont intitulés: "Sentiments du P. Claude Allouez sur les missions du

${ }^{7}$ Si le P. Dablon a adressé au Provincial de Paris d'autres notes du P. Allnnez. elles ne sont pas parvenues jusqu'à nous. 
Canada » et «Sentiments du P. Claude Allouez sur les missionnaires qu'il faut en Canada ». Sont-ils bien de lui ?

A notre avis, et c'est ce que nous prétendons établir, le $P$. Allouez, en un même document, avait uni aux Sentiments sur sa vocation aux missions des extraits capables de réchauffer sa ferveur et de l'aider à maintenir bien haut toujours son idéal missionnaire; c'était un vade-mecum, un aide-mémoire qui n'était pas destiné à la publication. Ce document lui était très précieux; et il est permis de croire qu'en ses trente années de vie dans les missions du Canada, il l'a non seulement relu très souvent, mais qu'il l'a plus d'une fois recopié ${ }^{8}$. Mais quelle est la source littéraire du P. Allouez?

\section{LA « RELATION 》 DE 1635}

Candidat aux missions de la Nouvelle-France, il était tout naturel que Claude Allouez cherchât à mieux connaître le champ de son futur apostolat, les peuples qu'il serait appelé à évangéliser, l'esprit dont il devait se revêtir dans les Relations des Jésuites de la Nouvelle-France. Or, il en était une qui répondait particulièrement aux aspirations de son âme. Il en fit des extraits qu'il copia à la suite de ses Sentiments sur sa vocation; le document n'était pas long. Mais par la richesse de son contenu, il valait toute une bibliothèque et serait pour Claude Allouez un aliment spirituel.

Nous n'avons pas à dire ici ce que sont les Relations des Jésuites. Il suffira de noter que celle de 1635 se terminait par des extraits empruntés à la correspondance personnelle des missionnaires, et que l'éditeur parisien avait ajoutés au manuscrit reçu de Québec. Cela s'intitulait: Divers sentiments et avis des Pères qui sont dans la Nouvelle-France. Tirés de leurs dernières lettres de $1635^{\circ}$. Trente et un extraits, dûment numé-

8 On expliquerait par là les variantes, le $P$. Allouez abrégeant le texte original, le modifiant un peu, liant parfois des phrases qui ne se suivent pas dans l'original.

9 Edition de Québec, Relation de 1635, 45-50; Thwaites, VIII: 168-192. Dans Jésuites de la Nouvelle-France (Collection Christus, Paris, Desclée De Brouwer), le P. François Roustang, S.J. a publié les « Divers sentiments et avis de 1635 », en les attribuant, par distraction, sans doute, selon nous, au P. Paul Le Jeune. 
rotés, d'une belle tenue littéraire et d'une très haute inspiration surnaturelle. S'adressant, non au grand public, mais à des âmes capables de les comprendre et de sympathiser avec leurs sentiments les plus intimes, les missionnaires parlent de l'abondance de leur cœur. Et comme leur cœur est grand, désireux de sainteté personnelle et de rayonnement apostolique, on a là comme un raccourci des problèmes que posent les missions du Canada, une indication de l'esprit qui animent les Pères, des moyens d'apostolat sur lesquels ils fondent leurs espoirs et le portrait du missionnaire idéal ${ }^{10}$. C'est la source littéraire des Sentiments du P. Allouez sur les missions du Canada et sur les missionnaires qu'il faut en Canada.

On remarquera, en effet, que les Sentiments du P. Allouez sont déjà contenus quant à la substance, et très souvent quant à la lettre dans les Divers sentiments de 1635 . Le P. Allouez a le mérite de les avoir groupés sous des titres. Il a parfois abrégé, il s'est parfois exprimé autrement: modifications verbales qui laissent intacte la substance du texte original. De même, il n'a rien ajouté de strictement personnel. Le document que nous livre le P. Dablon n'est pas le journal spirituel du P. Allouez, ni le lieu où il aurait résumé pour l'avantage des autres les résultats de sa longue expérience: c'est un programme, un plan de vie. Si le P. Allouez n'a pas le mérite d'avoir donné aux Sentiments leur expression littéraire, il a celui, combien plus grand ! d'y avoir inlassablement conformé sa vie et d'avoir laissé à ses amis, tel le P. Dablon, la conviction qu'il en était bel et bien l'auteur.

\section{CONCLUSION}

Le cas que nous venons d'étudier n'est pas en lui-même très important, il n'appartient pas à la grande histoire. Mais il ne laisse pas d'être instructif à plus d'un titre. Nous avons dit

10 Les Divers sentiments et avis sont anonymes. Mais nous connaissons les Pères qui étaient alors au Canada et qui pouvaient parler d'expérience. En voici la liste suivant leur date d'arrivée: 1632, saint Antoine Daniel, Paul Le Jeune, Anne de Noue, Ambroise Davost; 1633, saint Jean de Brébeuf et Enemond Massé; 1634, Julien Perrault, André Richard, Charles Lalemant et Jacques Buteux. 
ailleurs les heureux effets produits dans les âmes par la lecture des Relations des Jésuites ${ }^{11}$. Le P. Claude Allouez en est un exemple éloquent. Si les Divers sentiments et avis de 1635 ne sont pas à l'origine de sa vocation missionnaire - il avait 14 ans lors de leur publication et nous ne savons pas quand il les a lus pour la première fois -, ils ont été pour lui et jusqu'à la fin de sa vie un soutien, une force dans la poursuite de l'idéal qu'il s'était tracé aux heures ferventes de sa jeunesse.

En attribuant au P. Allouez des sentiments que celui-ci avait puisés ailleurs, le $\mathrm{P}$. Claude Dablon commettait une erreur. Mais qui osera lui jeter la pierre ? Il n'a manqué à aucune loi de la prudence ou de la charité chrétiennes; et peut-on en demander davantage à un homme de sa condition? Voyons comment les choses se sont passées. Le P. Dablon avait sous les yeux un document écrit tout entier de la main du P. Allouez. Le premier paragraphe commençait par ces mots: « Je reçus le 2 mars de cette année 1657 la permission d'aller en Canada »; cela était vrai du P. Allouez et n'était vrai que de lui. Tout le premier paragraphe d'ailleurs lui appartient. Pourquoi pas les deux autres ? Car ils sont de la même écriture, font partie d'un même document et ne présentent aucune indication de provenance étrangère.

Pour y voir clair, le P. Dablon aurait dû, et cela au moment même où il abordait le second paragraphe, avoir présentes à l'esprit les dernières pages de la Relation publiée en 1635, soit 54 ans plus tôt. Peut-on exiger d'un homme une si prodigieuse mémoire ? N'est-ce pas précisément pour en prévenir des défaillances que le P. Allouez avait copié et qu'il relisait souvent les Divers sentiments et avis de 1635 ? De plus, nous l'avons dit et nous le répétons, si le $\mathrm{P}$. Allouez n'est pas l'auteur des Sentiments, il en a été la vivante incarnation. Le P. Dablon n'avait aucune raison de lui en refuser la paternité. Son erreur ne le diminue pas à nos yeux; elle rappelle à l'historien que la vérité est parfois inaccessible même aux témoins les mieux placés pour savoir et les plus consciencieux. Quant à nous, les consta-

11 Etude sur les Relations des Jésuites (Montréal, 1940), 257 ss. 
tations que nous présentons ici, n'ont été ni prévues ni recherchées. Comme tant d'autres, nous avions déjà lu, mais en des temps différents, les Divers sentiments et avis de 1635 et les Sentiments $d u$ P. Allouez. La circonstance nouvelle, c'est qu'en relisant ceux-ci, nous avions présents à la mémoire ceux-là. Certaines expressions nous laissant l'impression du déjà lu ailleurs, nous avons voulu vérifier. Mais, nous étions loin de soupçonner jusqu'à quel point le P. Allouez avait emprunté à ses devanciers.

Il nous reste à comparer aux Divers sentiments de 1635 les Sentiments du P. Allouez. Nous datons ceux-ci de 1690, parce que c'est alors seulement qu'ils sont arrachés au secret qui les a longtemps enveloppés. A notre avis, le P. Allouez les a unis, pour la première fois, à ses Sentiments sur sa vocation, entre le 3 mars 1657 , date où sa vocation missionnaire est confirmée, et le 8 août 1665, date de son départ pour les missions outaouaises. Il n'est pas vraisemblable qu'il ait apporté là-bas la Relation de 1635.

La colonne de gauche reproduit, en indiquant les numéros qu'ils portent dans le texte original, les Divers sentiments et avis de 1635. Chaque extrait est suivi de la lettre $R$ et d'un chiffre: renvoi à la page de Jésuites de la Nouvelle-France du P. François Roustang (Collection Christus, Paris 1961, Desclée De Brouwer). La colonne de gauche présente fidèlement et intégralement les deux derniers paragraphes du P. Allouez, Sentiments sur les missions du Canada et Sentiments sur les missionnaires qu'il faut en Canada. Il résulte de la comparaison que le P. Allouez n'a pas copié tous les Divers sentiments; il en a fait un choix conforme aux aspirations et aux besoins de son âme. De plus, chaque paragraphe constitue un tout complet. La lettre $\mathrm{M}$ et le chiffre qui suit réfèrent à la page de Margry, Découvertes et Etablissements des Français (Paris, 1879), I: 64-72.

Ad complementum materix, nous publions d'abord le premier paragraphe du P. Allouez, en corrigeant les quelques erreurs de lecture de Margry.

LÉON POULIOT, s.j. 


\section{SENTIMENTS DU P. CLAUDE ALLOUEZ SUR SA VOCATION AUX MISSIONS}

Je recus le $3^{e}$ mars de cette année 1657 , la permission d'aller en Canada; je baisai aussitôt la lettre du Révérend Père Provincial, 12 et je me dis souvent à moi-même: "C'est le Seigneur qui me fait une si grande grâce; j'en suis dans l'étonnement et l'admiration. Après un si bon gage de sa bonté et de son amour envers moi, $j$ 'ai tout sujet de me flatter que je ne mourrai point; mais que je vivrai pour annoncer les merveilles de ses ouvres. C'est ici un coup de sa droite qui m'a exalté par la plus sublime de toutes les vocations. Seigneur, je suis à vous; secondez-moi dans cette divine entreprise, afin que je me sauve, et me sanctifie moi-même en travaillant au salut et à la sanctification du prochain. Mon âme vous loue, vous bénit, vous remercie et vous aime préférablement à tout ce qui n'est point vous. »

J'ai demandé et je chéris cette vocation, premièrement, Parce que c'est la plus grande gloire de Dieu que je me rende le plus semblable que je pourrai à Jésus-Christ, son Fils, qui, pouvant sauver les hommes sans souffrir, a, pour opérer ${ }^{13}$ leur salut, préféré la croix à la joie: Proposito sibi gaudio sustinuit crucem. ${ }^{14}$

Secondement, je ne dois pas regretter les douceurs que je quitte en sortant de France, et que j'aurais trouvées en mille et mille choses licites et honnêtes, comme entr'autres dans la conversation avec tant de personnes d'esprit, dans l'étude et la profession des plus belles sciences, et dans l'usage des talents que j'ai reçus du ciel. Non, non, je ne dois regretter rien de tout ce qu'il faut nécessairement quitter à la mort. Je veux prévenir ce temps de la séparation de mon âme d'avec mon corps, afin de laisser à présent, sans contrainte et avec mérite, ce qu'il me faudra laisser alors par force et sans liberté. D'ailleurs, tous les biens que je puis avoir ne m'ont été donnés de Dieu que pour en user selon son bon plaisir. Or il veut ${ }^{15}$ que ce soit en Canada que je m'en serve pour éclairer les nations auxquelles il m'envoie. Ad illuminationem gentium misit ${ }^{16}$ me. Je connais ses desseins

12 Margry: «Je laissai aussitôt la lettre du Révérend Père Provincial 》, ce qui n'a aucun sens. Il est tout naturel, au contraire, que le P. Allouez baise cette lettre qui lui manifeste une volonté de Dieu, si ardemment désirée. 13 Margry: «espérer », ce qui nous paraît une erreur de lecture. JésusChrist n'a pas souffert pour espérer, mais pour opérer le salut des hommes. 14 Margry a lu: Reposito sibi gaudio sustinuit crucem. Margry pouvait bien ignorer ce texte si connu de saint Paul; le P. Allouez ne l'ignorait certainement pas.

15 Margry a lu: il peut.

16 Margry: mittit me. 
sur moi par les fréquentes inspirations que j'ai là-dessus depuis si longtemps.

Troisièmement, je trouverai dans la Nouvelle-France la volonté de Dieu, la gloire de Notre-Seigneur, ma propre perfection et le salut des âmes avec beaucoup de souffrance. Si je suis assez malheureux que de n'y pas aller, c'est que mes péchés, et nommément mes péchés d'orgueil me rendent indigne d'une si sainte mission.

On m'allègue que je puis autant servir Dieu dans l'Ancienne France que dans la Nouvelle; que j'ai reçu du Ciel assez de talent pour les emplois de notre Institut, lesquels on exerce en Europe, et qu'en m'appliquant à la prédication, je pourrai sans beaucoup de peine m'entretenir dans une dévotion solide. J'avoue encore aux personnes qui me parlent ainsi, j'avoue que je découvre dans les missions du Canada des difficultés terribles à la nature. Mais dans tous ces prétextes et dans toutes ces oppositions, il n'y a rien qui ne me soit suspect d'amour-propre, et quand quelques pensées semblables se présentent à mon esprit, je me dis: "tu ne sais ce que tu crains! tu auras ton contentement dans la Nouvelle-France ». S'il arrivait donc que le Révérend Père Provincial voulût révoquer sa permission, je me jetterais à genoux à ses pieds pour le conjurer qu'il me la continuât.

Qui que ce soit qui prétende me détourner de ma chère mission, je lui répliquerai avec ces paroles de mon divin Maître: «Retire-toi de moi, Satan! Tu ne goûtes pas les choses de Dieu, mais tu parles en homme ». Pour moi, je ne puis plus vivre d'une vie tiède et imparfaite, après que notre Sauveur, qui m'a fait jusques ici tant de grâces, semble y avoir mis le comble par la grâce de ma vocation en Canada: on attend de moi plus de ferveur et de fidélité que je n'en ai eu jusqu'à présent, et l'on serait scandalisé de moi, si dans cette occasion, je montrais de la lâcheté ou de l'inconstance.

\section{SENTIMENTS DU P. CLAUDE ALLOUEZ SUR LES MISSIONS DU CANADA}

Divers sentiments et avis (1635)

1. La Nouvelle-France est un vrai climat où on apprend parfaitement bien à ne chercher

\author{
Sentiments \\ du P. Claude Allouez (1690) \\ La Nouvelle-France est une éco- \\ le où l'on apprend en perfec- \\ tion à ne chercher que Dieu, à
}


que Dieu, ne désirer que Dieu seul, avoir l'intention purement à Dieu, et à ne s'attendre et ne s'appuyer qu'en sa divine et paternelle providence. R. 72 .

2. Vivre en la Nouvelle-France, c'est à vrai dire, vivre dans le sein de Dieu, et ne respirer que l'air de sa divine conduite. On ne saurait croire la douceur de cet air-là, si ce n'est quand actuellement on le respire. $R$. 72.

3. Il n'est pas à propos que tout le monde sache combien il fait bon dans les sacrées horreurs de ces forêts, et combien on trouve de lumières du ciel dans les ténèbres épaisses de cette barbarie. Nous aurions trop de monde qui y voudrait venir, et nos habitations ne seraient pas capables de loger tant de gens; et c'est ce qui nous confond que Dieu nous ait choisis pour nous faire participants de cette miséricorde, voyant qu'il y a tant de nos Pères en France qui feraient mieux que nous. R. 72 .

4. La joie qu'on a, quand on a baptisé un sauvage, qui se meurt peu après et qui s'envole droit au ciel pour devenir un ange, certainement c'est une joie qui surpasse tout ce qu'on ne désirer que Dieu; à n'avoir son intention qu'à Dieu, et à ne s'attendre et à ne s'appuyer que sur la toute paternelle providence de Dieu. M. 68.

Vivre dans les missions du Canada, c'est à vrai dire vivre dans le sein de la Divinité et ne respirer que l'air de sa divine conduite. On ne saurait croire combien cet air est doux, si ce n'est lorsqu'on le respire. M. 68.

Il n'est pas à propos que tout le monde sache quelle est sa douceur, et qu'elle est de travailler au salut des âmes. ${ }^{17} \mathrm{Oh}$ ! qu'il fait bon dans les sacrées horreurs de ces forêts. Oh ! qu'on trouve de lumières dans les épaisses ténèbres de cette barbarie; nous aurions trop de monde qui y voudrait venir, si le bonheur que nous avons d'y être était plus connu. $\mathrm{Ah}$ ! c'est ce qui nous confond que Dieu nous ait choisis pour nous rendre participants d'une grâce si signalée, quoiqu'il y ait en France tant de nos Pères qui feraient mieux ici que nous. M. 68.

En vérité, la Nouvelle-France est un pays de douceur; et elle le serait même quand il n'y en aurait point d'autre que la joie qu'on sent d'avoir baptisé un sauvage, lequel mourant peu

17 « et quelle la douceur de travailler au salut des âmes », phrase intelligible, mais un peu déroutante. Dans une autre copie des Sentiments, dont nous ignorons la provenance, on lit ici : * et quel bien c'est d'y travailler au salut des âmes ». 
se peut imaginer; on ne se souvient plus ni de la mer, ni du mal de la mer, ni de l'horreur des tempêtes passées; on voudrait avec la souffrance de dix mille tempêtes pouvoir aider à sauver une âme, puisque JésusChrist pour une seule âme aurait volontiers répandu tout son précieux sang. R. 72 .

5. ...... Une fois qu'on a goûté à bon escient la douceur de la croix de Jésus-Christ, on la préfère à tous les empires de la terre. R. 73 .

8. Jamais je n'avais entendu que c'est d'arriver à un point de vertu, que pour passer plus avant il faudrait faire un miracle. Tant il est vrai qu'on se trouve quelquefois si avant dans les souffrances, ou dans les hasards, ou dans l'abandonnement des créatures, qu'on ne trouve plus rien que Dieu. Mais on le trouve toujours au bout de l'échelle de Jacob, à bras et à cœur ouverts pour embrasser les anges et les âmes qui volent droit à lui; et c'est chose admirable comme Dieu prend plaisir à se communiquer abondamment aux âmes qui ont tout abandonné et se sont toutes abandonnées à lui. Perdre tout pour trouver Dieu, c'est une douce perte et une sainte usure. R. 74 .

7. ..... mais on ne croirait jamais l'efficace de la grâce et les puissantes assurances que Dieu donne à ses serviteurs, au mi- après son baptême, s'en va droit au ciel. Cette joie surpasse tout ce qu'on peut s'imaginer de joie en ce monde. On ne se souvient plus des travaux passés, et l'on voudrait souffrir mille fois plus s'il était nécessaire pour obtenir encore le salut de quelque âme. M. 68.

Qui a goûté la douceur de JésusChrist, la préfère même aux empires de la terre. M. 69.

La Nouvelle-France ne laisse pas d'être aussi un lieu de souffrance. On y est quelquefois éprouvé par tant de peines, épuisé de si rudes travaux, environné de si grands dangers, et tellement abandonné des créatures que l'on ne trouve que Dieu seul; mais on le trouve au bout de l'échelle, tel qu'il se montra à Jacob, les bras étendus et le cœur ouvert pour recevoir les âmes généreuses qui comme autant d'anges visibles, volent droit au ciel. C'est chose admirable comment Dieu prend plaisir à se communiquer abondamment aux personnes qui, ayant abandonné tout pour son amour, sont entièrement abandonnées à lui. Perdre tout pour trouver Dieu, c'est une heureuse perte et une sainte usure. M. 69.

On ne le croirait jamais, si on ne l'avait éprouvé, combien sont abondantes les grâces, combien puissantes sont les as- 
lieu des tempêtes et des désespoirs les plus épouvantables. R. 73-74.

9. Le cœur croît à mesure que les travaux croissent pour Jésus-Christ; et la NouvelleFrance est le pays du monde le plus propre pour entendre le sens littéral de ces belles paroles, Sicut misit me vivens Pater, ita et ego mitto vos, je vous envoie de la même sorte que mon Père m'a envoyé: Ecce ego mitto vos sicut oves in medio luporum. Voici que je vous envoie comme des brebis au milieu des loups. Parmi ces forêts, en voyant ces Sauvages, nous, pauvres étrangers et serviteurs de Dieu, que pouvonsnous attendre sinon un coup de dent et quelque effet de leur barbarie naturelle ? Qui craint bien Dieu ne saurait plus rien craindre en ce monde. R. 74 .

13. Trois puissantes pensées consolent un bon cœur, qui est dans les forêts infinies de la Nouvelle-France ou parmi les Hurons. La première est: « Je suis où Dieu m'a envoyé, où il m'a mené comme par la main, où il est avec moi, et où je ne cherche que lui seul ». La deuxième est ce que dit David: «Selon la mesure des douleurs que je souffre pour Dieu, ses divines consolations réjouissent mon âme ». La troisième, que jamais on ne trouve ni croix, ni clous, ni épines, que, si on re- surances que le Seigneur donne à ses serviteurs au milieu même des désespoirs les plus épouvantables. ${ }^{18}$ M. 69.

Le cœur croît à proportion que croissent les travaux pour Jésus-Christ. La Nouvelle-France est le pays du monde le plus propre à concevoir le sens littéral de ces paroles du Sauveur: «Voilà que je vous envoie comme mon Père m'a envoyé, en vous envoyant comme des brebis au milieu des loups ». En effet, il nous envoie dans de vastes forêts, parmi des Sauvages si cruels qu'ils s'entremangent les uns les autres. Qu'en devons nous donc attendre, sinon des coups de dents, ou des effets encore plus horribles de leur barbarie? Mais ne craignez point ceux qui ne peuvent tuer que le corps. M. 69.

Trois pensées consolent un bon cœur au milieu des dangers, des travaux et des peines où il se trouve dans nos missions du Canada. La première de ces pensées est celle-ci: je suis où Dieu me veut, où il m'a envoyé, où il m'a conduit comme par la main, où il est avec moi et où je ne cherche que lui. La deuxième est que plus j'aurai à souffrir, plus j'aurai de consolation. Secundum multitudinem dolorum meorum, consolationes tuæ lætificaverunt animam meam. La troisième est qu'on

18 Phrase ainsi soulignée dans Margry. 
garde bien, on ne trouve JésusChrist au milieu. Or, peut-on être mal quand on est en compagnie du Fils de Dieu vivant ? R. 75 .

14. Quand je me vois assiégé de flots homicides, de forêts infinies et de mille dangers, il me vient à l'esprit cette riche parole de saint Ignace, martyr: Nunc incipio esse Christi discipulus, "C'est aujourd'hui que je commence d'être de la Compagnie de Jésus », car à quoi servent tant d'exercices, tant de méditations ferventes, tant de désirs bouillants ? Tout cela n'est que du vent, si on ne les met en pratique; tellement que la vieille France est bonne pour concevoir de bons désirs, mais la Nouvelle est propre pour l'exécution; ce que l'on désire en l'ancienne France, c'est ce qu'on fait dans la Nouvelle. R. 76 .

16. ..... je ne crois pas qu'on puisse mieux vivre, ni avec plus de satisfaction et de courage, et même de mérites, que vivre en un lieu où on pense pouvoir mourir tous les jours, et avoir la devise de S. Paul: Quotidie morior, fratres, etc. ; « mes frères, je fais état de mourir tous les jours ». R. 76 .

10. ..... les infusions de Dieu dans les cœurs et le renfort qu'il verse dans nos âmes surpassent tous nos maux. R. 74 .

15. ..... quel bonheur de ne voir rien de ses yeux que des ne trouve jamais de croix, de clous et d'épines qu'on ne trouve Jésus au milieu. M. 69-70.

Quand je me vois assiégé des flots, dans une tempête, ou exposé à d'autres dangers, je me souviens de celui qui disait en ces semblables rencontres : c'est à présent que je commence à être disciple de Jésus-Christ. L'Ancienne France est bonne pour concevoir de fervents désirs, et la Nouvelle pour les exécuter. M. 70.

Un missionnaire du Canada prend cette devise de saint Paul: «Je meurs tous les jours ». M. 70.

mais aussi les grâces que Dieu verse dans nos cœurs surpassent tous nos maux. M. 70 .

Quel bonheur pour nous de ne voir ici que des croix, des Sau- 
Sauvages, des croix et JésusChrist! En ma vie je n'ai bien compris en France ce que c'était de se défier totalement de soimême et se confier en Dieu seul. Je dis seul et sans mélange d'aucune créature. R. 76 .

28. La pensée de saint Francois-Xavier nous passe mille fois par l'esprit et a un grand pouvoir. Si les hommes du siècle, pour avoir des peaux de castor, de la morue et je ne sais quelles denrées, n'appréhendent ni les orages de la mer, ni les Sauvages de la terre, ni la mer, ni la mort; quelle horrible confusion serait-ce à des serviteurs de Dieu, d'appréhender cela ou quelques petits travaux, pour tâcher de gagner des âmes rachetées avec le sang précieux de Jésus-Christ et empourprées de son sang de valeur inestimable! R. 80 . vages et Jésus-Christ. Je n'avais jamais compris en Europe ce que c'est, que de se défier entièrement de soi-même et de se confier totalement en Dieu seul. Je dis seul, et sans mélange d'aucune pure créature. M. 70.

La pensée de saint FrançoisXavier en est très puissante sur mon esprit. Si les hommes du siècle s'exposent à tant de périls et entreprennent de si grands travaux pour gagner des biens périssables, quelle confusion serait-ce à des serviteurs de Dieu d'appréhender les peines et les dangers pour gagner des âmes rachetées du précieux sang de Jésus-Christ. M. 70 .

\section{SENTIMENTS DU P. CLAUDE ALLOUEZ SUR LES MISSIONNAIRES QU'IL FAUT EN CANADA}

\section{Divers sentiments et avis}

(1635)

30. L'expérience nous fait voir que ceux de la Compagnie qui viennent en la Nouvelle-France, il faut qu'ils y soient appelés par une vocation spéciale et bien forte; que ce soit gens morts à soi et au monde, hommes véritablement apostoliques, qui ne cherchent que Dieu et

\begin{abstract}
Sentiments
du P. Claude Allouez (1690)

Les religieux de la Compagnie de Jésus qui passent de l'Ancienne France à la Nouvelle doivent y être appelés par une spéciale et forte vocation. Il faut qu'ils soient des gens morts au monde et à eux-mêmes, des hommes apostoliques et des saints qui ne cherchent que
\end{abstract}


le salut des âmes, qui aiment d'amour la croix et la mortification; qui ne s'épargnent point; qui sachent supporter les travaux de la mer et de la terre, et qui désirent plus la conversion d'un Sauvage que l'empire de toute l'Europe; qui aient des cours de Dieu et tout remplis de Dieu, qui soient comme des petits Jean-Baptiste, criant parmi ces déserts et ces forêts, comme des voix de Dieu, qui appellent tous ces pauvres Sauvages à reconnaître JésusChrist; enfin, que ce soit des hommes qui ont tous leurs contentements dans Dieu, et auxquels les souffrances soient leurs plus chères délices. Voilà ce que l'expérience nous fait voir tous les jours. Mais aussi, il est vrai qu'il semble que Dieu répande bien plus abondamment les rosées de ses grâces sur cette Nouvelle-France que sur la vieille, et que les consolations intérieures et les divines infusions y sont bien plus solides, et les cœurs bien plus embrasés. Novit Dominus qui sunt ejus. Mais il n'appartient qu'à Dieu de faire le choix de ceux dont il veut se servir, et auxquels il fait cette miséricorde de les amener en la NouvelleFrance pour en faire des saints. Saint François-Xavier disait qu'il y avait une île en Orient, qui était bien propre pour faire perdre la vue, à force de pleurer de joie excessive du cœur; je ne sais si notre Nouvelle-France ressemble point cette île; mais nous expérimentons que si quel-
Dieu et le salut des âmes. Il faut qu'ils aiment d'amour la croix et la mortification; qu'ils ne s'épargnent point, qu'ils sachent supporter les travaux de la mer et de la terre, et qu'ils désirent plus la conversion d'un sauvage qu'un empire. Il faut qu'ils soient dans les forêts du Canada comme autant de précurseurs de Jésus-Christ et que, comme des petits Jean-Baptiste, ils soient autant de voix de Dieu lesquelles crient dans les déserts pour appeler les sauvages à la connaissance du Sauveur. Enfin, il faut qu'ils aient mis tout leur appui et leur contentement et leur trésor en Dieu seul à qui seul il appartient de choisir ce qu'il veut pour le Canada. Novit Dominus qui ejus sunt. Il leur fait cette miséricorde pour en faire des saints. S'ils sont fidèles à leur vocation, ils éprouvent que si, comme saint Xavier l'assure, il y a une île en Orient, où à force de pleurer de joie, on est pour perdre les yeux; de même en s'abandonnant à Dieu tout de bon en Canada, on y perdra avec joie la vue, la santé et la vie à force d'y travailler et d'y souffrir pour la conversion des Sauvages à la gloire de JésusChrist. M. 71-72. 
qu'un ici s'abandonne à Dieu à bon escient, il court hasard d'y perdre la vue, et la vie, et tout, et avec grande joie, à force de travailler; il n'appartient qu'à ceux qui y sont et qui goûtent Dieu d'en parler par expérience. R. 80-81.

17. Pour convertir les Sauvages, il n'y faut pas tant de science que de bonté et de vertu bien solide. Les quatre éléments d'un homme apostolique en la Nouvelle-France sont l'affabilité, l'humilité, la patience et une charité généreuse. Le zèle trop ardent brûle plus qu'il n'échauffe et gâte tout; il faut une grande magnanimité et condescendance pour attirer peu à peu ces Sauvages. Ils n'entendent pas bien notre théologie, mais ils entendent parfaitement bien notre humilité et notre affabilité, et se laissent gagner. $\mathrm{R}$. 77.

24. J'avais cru qu'il fallait des miracles pour convertir ces Sauvages volants; mais je me suis trompé, car les miracles propres de la Nouvelle-France sont ceux-ci : leur faire du bien et souffrir bien des maux, ne s'en plaindre qu'à Dieu, s'en estimer indigne, et se tenir pour fort inutile. R. 78 .

21. On dit que les premiers qui fondent les Eglises d'ordinaire sont saints; cette pensée m'attendrit si fort le cœur que, quoique je me voie ici fort inutile dans cette fortunée Nouvelle-
Pour convertir les Sauvages, il ne faut pas tant de science que de sainteté. Un zèle trop ardent y gâte tout; leur naturel, froid et indifférent, ne veut pas être si vivement pressé. S'il m'est permis de m'exprimer ainsi ; les quatre éléments d'un homme apostolique, en la NouvelleFrance, sont: la condescendance et l'affabilité, la force et la patience avec une charité et magnanimité héroïques. M. 72.

Pour convertir nos sauvages et barbares ou nos Sauvages du Canada, il n'est point besoin d'autres miracles que de leur faire du bien, de souffrir beaucoup, de ne se plaindre de ses peines qu'à Dieu et de se tenir pour un serviteur inutile. M. 72.

On dit que les premiers qui fondent des Eglises sont ordinairement des saints; cette pensée m'attendrit tellement le cœur que, quoique je ne sois bon à rien, je désire me consumer de 
France, si faut-il que j'avoue plus en plus pour la salut des que je ne saurais me défendre âmes. Cupio 19 impendi et sud'une pensée qui me presse le perimpendi pro animabus. M. cœur: Cupio impendi et super- 72.

impendi pro vobis. R. 78.

19 Margry : « Cupidè impendi ...»

A ceux de nos abonnés possédant les numéros des volumes I-III-V-XI-XIII et XIV dont ils voudraient bien disposer, prière de communiquer avec le secrétariat (261 avenue Bloomfield, Outremont 8. Tél.: CR. 1-8264). Nous en aurions un urgent besoin pour répondre à la demande de collections complètes. 